\title{
SUBMODULES OF SPECHT MODULES FOR WEYL GROUPS
}

\author{
by SAITT HALICIOĞLU
}

(Received 22nd March 1994)

\begin{abstract}
The construction of all irreducible modules of the symmetric groups over an arbitrary field which reduce to Specht modules in the case of fields of characteristic zero is given by $G$. D. James. Halicioğlu and Morris describe a possible extension of James' work for Weyl groups in general, where Young tableux are interpreted in terms of root systems. In this paper we show how to construct submodules of Specht modules for Weyl groups.
\end{abstract}

1991 Mathematics subject classification: Primary 20 C33 and 20F55, Secondary 22E45.

\section{Introduction}

The representation theory of the symmetric groups over fields of characteristic zero is well developed and documented. The original approach was due to G. Frobenius and I. Schur followed independently by A. Young. In the 1930's, W. Specht [8] presented an alternative approach to give a full set of irreducible modules now called Specht modules. In 1976, G. D. James, in a very important paper [6], introduced an easy and ingenious construction of all the irreducible modules of the symmetric groups over an arbitrary field which reduce to Specht modules in the case of characteristic zero. Al-Aamily, Morris and Peel [1] showed how this construction could be extended to deal with the Weyl groups of type $B_{n}$. In [7] Morris described a possible extension of James' work for Weyl groups in general. An alternative and improved approach was described by the present author and Morris [5]. Later on the present author [4] develop the theory and show how a $K$-basis for Specht modules can be constructed in terms of standard tabloids and introduce an algorithm which results a basis for a Specht modules for Weyl groups in many cases. Recently, a possible extension of James' work for finite reflection groups has been given in [3]. In this paper we further develop the theory and show how to construct submodules of Specht modules for Weyl groups.

\section{Notation and preliminary results}

In this section we establish the notation and state preliminary results on Specht modules for Weyl groups. The basic definitions and background material required here may be found in [5].

2.1 Let $\boldsymbol{V}$ be $l$-dimensional Euclidean space over the real field $\mathbf{R}$ equipped with a positive definite inner product (,). For $\alpha \in V, \alpha \neq 0$, let $\tau_{\alpha}$ be the reflection in the hyperplane orthogonal to $\alpha$, that is, $\tau_{\alpha}$ is the linear transformation on $V$ defined by 


$$
\tau_{\alpha}(v)=v-2 \frac{(\alpha, v)}{(\alpha, \alpha)} \alpha
$$

for all $v \in V$. Let $\Phi$ be a root system in $V$ and $\pi$ be a simple system in $\Phi$ with corresponding positive system $\boldsymbol{\Phi}^{+}$and negative system $\boldsymbol{\Phi}^{-}$. Then, the Weyl group of $\Phi$ is the finite reflection group $\mathscr{W}=\mathscr{W}(\Phi)$ which is generated by the $\tau_{a}, \alpha \in \Phi$.

If $\boldsymbol{\Psi}$ is a subsystem of $\boldsymbol{\Phi}$ with simple system $\boldsymbol{J} \subset \boldsymbol{\Phi}^{+}$and Dynkin diagram $\Delta$ and let $\boldsymbol{\Psi}=\bigcup_{i=1}^{i} \boldsymbol{\Psi}_{i}$, where $\boldsymbol{\Psi}_{i}$ are the indecomposable components of $\boldsymbol{\Psi}$. Let $J_{i}$ be a simple system in $\Psi_{i}(i=1,2, \ldots, r)$ and $J=\bigcup_{i=1}^{r} J_{i}$. Let $\Psi^{\perp}$ be the largest subsystem in $\Phi$ orthogonal to $\boldsymbol{\Psi}$ and let $J^{\perp} \subset \Phi^{+}$be the simple system of $\Psi^{\perp}$. If $\Psi^{\prime}$ is a subsystem of $\Phi$ which is contained in $\boldsymbol{\Phi} \backslash \Psi$, with simple system $J^{\prime} \subset \Phi^{+}$and Dynkin diagram $\Delta^{\prime}$, $\boldsymbol{\Psi}^{\prime}=\bigcup_{i=1}^{s} \boldsymbol{\Psi}_{i}^{\prime}$, where $\boldsymbol{\Psi}_{i}^{\prime}$ are the indecomposable components of $\boldsymbol{\Psi}^{\prime}$ then let $J_{i}^{\prime}$ be a simple system in $\Psi_{i}^{\prime}(i=1,2, \ldots, s)$ and $J^{\prime}=\bigcup_{i=1}^{s} J_{i}^{\prime}$. Let $\Psi^{\prime \perp}$ be the largest subsystem in $\Phi$ orthogonal to $\Psi^{\prime}$ and let $J^{\perp} \subset \Phi^{+}$be the simple system of $\Psi^{\prime \perp}$. If $\bar{J}$ stand for the ordered set $\left\{J_{1}, J_{2}, \ldots, J_{r} ; J_{1}^{\prime}, J_{2}^{\prime}, \ldots, J_{s}^{\prime}\right\}$, where in addition the elements in each $J_{i}$ and $J_{i}^{\prime}$ are also ordered, then let $\Upsilon_{\Delta}=\{w \bar{J} \mid w \in \mathscr{W}\}$. The pair $\bar{J}=\left\{J, J^{\prime}\right\}$ is called a useful system in $\Phi$ if $\mathscr{W}(J) \cap \mathscr{W}\left(J^{\prime}\right)=\langle e\rangle$ and $\mathscr{W}\left(J^{\perp}\right) \cap \mathscr{W}\left(J^{\prime}\right)=\langle e\rangle$. The elements of $\Upsilon_{\Delta}$ are called $\Delta$-tableaux, the $J_{i}$ and $J_{i}^{\prime}$ are called the rows and the columns of $\left\{J, J^{\prime}\right\}$ respectively. Two $\Delta$-tableaux $\bar{J}$ and $\bar{K}$ are row-equivalent, written $\bar{J} \sim \bar{K}$, if there exists $w \in \mathscr{W}(J)$ such that $\bar{K}=w \bar{J}$. The equivalence class which contains the $\Delta$-tableau $\bar{J}$ is denoted by $\{\bar{J}\}$ and is called a $\Delta$-tabloid. Let $\tau_{\Delta}$ be set of all $\Delta$-tabloids. Then $\tau_{\Delta}=\left\{\{\overline{d J}\} \mid d \in D_{\Psi}\right\}$, where $D_{\Psi}=\left\{w \in \mathscr{W} \mid w(j) \in \Phi^{+}\right.$for all $\left.j \in J\right\}$ is a distinguished set of coset representatives of $\mathscr{W}(\Psi)$ in $\mathscr{W}$. The group $\mathscr{W}$ acts on $\tau_{\Delta}$ as $\sigma\{\overline{w J}\}=\{\overline{\sigma w J}\}$ for all $\sigma \in \mathscr{W}$. Let $K$ be arbitrary field, let $M^{\Delta}$ be the $K$-space whose basis elements are the $\Delta$-tabloids. Extend the action of $\mathscr{W}$ on $\tau_{\Delta}$ linearly on $M^{\Delta}$, then $M^{\Delta}$ becomes a $K \mathscr{W}$-module. Let

$$
\kappa_{J^{\prime}}=\sum_{\sigma \in W\left(J^{\prime}\right)} s(\sigma) \sigma \text { and } e_{J, J^{\prime}}=\kappa_{J^{\prime}}\{\bar{J}\}
$$

where $s(\sigma)=(-1)^{l(\sigma)}$ is the sign function and $l(\sigma)$ is the length of $\sigma$. Then $e_{J, J^{\prime}}$ is called the generalized $\Delta$-polytabloid associated with $\bar{J}$. Let $S^{J, J^{\prime}}$ be the subspace of $M^{\Delta}$ generated by $e_{w J, w J^{\prime}}$ where $w \in \mathscr{W}$. Then $S^{J, J^{\prime}}$ is called a generalized Specht module. A useful system $\left\{J, J^{\prime}\right\}$ in $\Phi$ is called a good system if $d \Psi \cap \Psi^{\prime}=\emptyset$ for $d \in D_{\Psi}$ then $\{\overline{d J}\}$ appears with non-zero coefficient in $e_{J, J^{\prime}}$. If $\left\{J, J^{\prime}\right\}$ is a good system, then $S^{J, J^{\prime}}$ is irreducible, [5].

2.2 Let $\boldsymbol{\Psi}$ be a subsystem of $\boldsymbol{\Phi}$ with simple $J$. If $\mathscr{N}(\Psi)=\{w \in \mathscr{W} \mid w \boldsymbol{\Psi}=\boldsymbol{\Psi}\}$ and $\mathscr{N}(J)=\{w \in \mathscr{W} \mid w(J)=J\}$, then $\mathscr{N}(\Psi)=\mathscr{W}(J) \times \mathscr{N}(J)$, where $\times$ denotes semidirect product [2].

\section{Submodules of Specht modules for Weyl groups}

In this section we show how to construct submodules of Specht modules for Weyl groups. Detailed proofs are not always included as they are either given or modifications of those in the earlier paper [5]. 
Let $\bar{J}$ be the ordered set $\left\{J_{1}, J_{2}, \ldots, J_{r} ; J_{1}^{\prime}, J_{2}^{\prime}, \ldots, J_{s}^{\prime}\right\}$, satisfying (2.1) and let

$$
\Upsilon_{\Delta}=\{w \bar{J} \mid w \in \mathscr{W}\} .
$$

Now we can give our principal definition.

Definition 3.1. Let $\Psi$ and $\Psi^{\prime}$ be subsystems of $\Phi$ with simple systems $J$ and $J^{\prime}$ respectively such that $\Psi^{\prime} \subseteq \Phi \backslash \Psi$ and $J \subset \Phi^{+}, J^{\prime} \subset \Phi^{+}$. The pair $\left\{J, J^{\prime}\right\}$ is called a useful sub-system in $\Phi$ if $\mathscr{N}(\Psi) \cap \mathscr{W}\left(\Psi^{\prime}\right)=\langle e\rangle$ and $\mathscr{W}\left(\Psi^{\perp}\right) \cap \mathscr{W}\left(\Psi^{\prime \perp}\right)=\langle e\rangle$.

Remark 1. If $\left\{J, J^{\prime}\right\}$ is a useful sub-system in $\Phi$ then $\left\{J, J^{\prime}\right\}$ is a useful system in $\Phi$. If $\mathscr{N}(J)=\langle e\rangle$, then the converse is true.

Definition 3.2. Let $\left\{J, J^{\prime}\right\}$ be a useful sub-system in $\Phi$. Then the elements of $\Upsilon_{\Delta}$ are called $\Delta$-tableaux.

Definition 3.3. Two $\Delta$-tableaux $\bar{J}$ and $\bar{K}$ are row-equivalent, written $\bar{J} \sim \bar{K}$, if there exists $w \in \mathcal{N}(\Psi)$ such that $\vec{K}=w \bar{J}$. The equivalence class which contains the $\Delta$-tableau $\bar{J}$ is $\{\bar{J}\}$ and is called a $\Delta^{*}$-tabloid.

Let $\tau_{\Delta^{*}}$ be the set of all $\Delta^{*}$-tabloids. It is clear that the number of distinct elements in $\tau_{\Delta^{*}}$ is $[\mathscr{W}: \mathcal{N}(\Psi)]$ and if $E_{\Psi}$ is the set of left coset representatives of $\mathcal{N}(\Psi)$ in $\mathscr{W}$, then we have

$$
\tau_{\Delta^{*}}=\left\{\{d \bar{J}\} \mid d \in E_{\boldsymbol{\Psi}}\right\}
$$

We note that since $\mathscr{W}(\Psi)$ is a subgroup of $\mathscr{N}(\Psi)$, then $E_{\Psi} \subseteq D_{\Psi}$ and if $\bar{J}=\left\{J ; J^{\prime}\right\}$, then $d J \subset \Phi^{+}$but $d J^{\prime}$ need not be a subset of $\Phi^{+}$.

We now give an example to show how to construct a $\Delta^{*}$-tabloid. In this example and later examples we use the following notation. If $\pi=\left\{\alpha_{1}, \alpha_{2}, \ldots, \alpha_{n}\right\}$ is a simple system in $\Phi$ and $\alpha \in \Phi$, then $\alpha=\sum_{i=1}^{n} a_{i} \alpha_{i}$, where $a_{i} \in \mathbf{Z}$. From now on $\alpha$ is denoted by $a_{1} a_{2} \ldots a_{n}$ and $\tau_{\alpha_{1}}, \tau_{\alpha_{2}}, \ldots, \tau_{\alpha_{n}}$ are denoted by $\tau_{1}, \tau_{2}, \ldots, \tau_{n}$ respectively.

Example 3.4. Let $\boldsymbol{\Phi}=\mathbf{A}_{3}$ with simple system $\pi=\left\{\alpha_{1}=\varepsilon_{1}-\varepsilon_{2}, \alpha_{2}=\varepsilon_{2}-\varepsilon_{3}, \alpha_{3}=\varepsilon_{3}-\varepsilon_{4}\right\}$. Let $\boldsymbol{\Psi}=\mathbf{2 A _ { 1 }}$ be the subsystem of $\Phi$ with simple system $J=\{100,001\}$. Let $\boldsymbol{\Psi}^{\prime}=\mathbf{A}_{1}$ be the subsystem of $\Phi$ which is contained in $\Phi \backslash \Psi$, with simple system $J^{\prime}=\{110\}$. Since $\mathscr{N}(\Psi) \cap \mathscr{W}\left(\Psi^{\prime}\right)=\langle e\rangle$ and $\mathscr{W}\left(\Psi^{\perp}\right) \cap \mathscr{W}\left(\Psi^{\prime \perp}\right)=\langle e\rangle$, then $\left\{J, J^{\prime}\right\}$ is a useful sub-system in $\Phi$.

Then $\tau_{\Delta^{*}}$ contains the $\Delta^{*}$-tabloids;

$$
\begin{aligned}
& \{\bar{J}\}=\{100,001 ; 110\} \\
& \left\{\tau_{2} \bar{J}\right\}=\{110,011 ; 100\} \\
& \left\{\tau_{1} \tau_{2} \bar{J}\right\}=\{010,111 ; 100\} .
\end{aligned}
$$


The group $\mathscr{W}$ acts on $\tau_{\Delta^{*}}$ according to

$$
\sigma\{\overline{w J}\}=\{\overline{\sigma w J}\} \quad \text { for all } \sigma \in \mathscr{W} .
$$

This action is easily seen to be well defined.

Now let $K$ be an arbitrary field and let $M^{\Psi}$ be the $K$-space whose basis elements are the $\Delta^{*}$-tabloids. Extend the action of $\mathscr{W}$ on $\tau_{\Delta}^{*}$ linearly on $M^{\psi}$, then $M^{\Psi}$ becomes a $K \mathscr{W}$-module. Then the $K \mathscr{W}$-module $M^{\boldsymbol{\Psi}}$ is a cyclic $K \mathscr{W}$-module generated by any one tabloid and $\operatorname{dim}_{K} M^{\Psi}=[\mathscr{W}: \mathcal{N}(\Psi)]$. Since $E_{\boldsymbol{\Psi}} \subseteq D_{\Psi}$, then $M^{\boldsymbol{\Psi}}$ is a $K \mathscr{W}$-submodule of $M^{\Delta}$.

Now we proceed to consider the possibility of constructing a $K \mathscr{W}$-module which corresponds to the Specht module in the case of symmetric groups. In order to do this we need to define a $\Delta^{*}$-polytabloid.

Definition 3.5. Let $\left\{J, J^{\prime}\right\}$ be a useful sub-system in $\boldsymbol{\Phi}$. Let

$$
\kappa_{J^{\prime}}=\sum_{\sigma \in W_{\left(\mathbf{\Psi}^{\prime}\right)}} s(\sigma) \sigma \text { and } e_{J, J^{\prime}}=\kappa_{J^{\prime}}\{\bar{J}\}
$$

where $s$ is the sign function. Then $e_{J, J}$ is called the generalized $\Delta^{*}$-polytabloid.

If $w \in \mathscr{W}$, then $w e_{J, J^{\prime}}=\kappa_{w J^{\prime}}\{\overline{w J}\}=e_{w J, w J^{\prime}}$. Hence, if $S^{\Psi, \Psi^{\prime}}$ is the subspace of $M^{\Psi}$ generated by $e_{w J, w J^{\prime}}$ where $w \in \mathscr{W}$, then $S^{\Psi^{\prime} \Psi^{\prime}}$ is a $K \mathscr{W}$-submodule of $M^{\Psi}$ and $S^{\Delta, \Delta^{\prime}}$, which is called a submodule of generalized Specht module. The $K \mathscr{W}$-module $S^{\Psi, \Psi}$ is a cyclic submodule generated by any $\Delta^{*}$-polytabloid.

The submodule of Specht module is spanned by the $e_{w J, w J}$ for all $w \in \mathscr{W}$. But it is easy to show that $S^{\Psi, \Psi^{\prime}}$ is generated by $e_{d J, d J^{\prime}}$, where $d \in D_{\Psi^{\prime}}$. Now we can give the following lemma.

Lemma 3.6. Let $\left\{J, J^{\prime}\right\}$ be a useful sub-system in $\Phi$ and let $d \in E_{\Psi}$. If $\{\overline{d J}\}$ appears in $e_{J . J}$ then it appears only once.

Proof. If $\sigma, \sigma^{\prime} \in \mathscr{W}\left(\Psi^{\prime}\right)$ and suppose that $\sigma=d w, \sigma^{\prime}=d w^{\prime}$ where $w, w^{\prime} \in \mathcal{N}(\Psi)$. Then $d=\sigma w^{-1}=\sigma^{\prime} w^{\prime-1}$ and $\sigma^{\prime-1} \sigma=w^{\prime-1} w \in \mathscr{N}(\Psi) \cap \mathscr{W}\left(\Psi^{\prime}\right)=\langle e\rangle$. Hence we have $w=w^{\prime}$ and $\sigma=\sigma^{\prime}$. Then $\{\overline{d J}\}$ appears in $e_{J, J^{\prime}}$ only once.

Corollary 3.7. If $\left\{J, J^{\prime}\right\}$ is a useful sub-system in $\Phi$, then $e_{J, J^{\prime}} \neq 0$.

Proof. By Lemma 3.6 if $\{\overline{\sigma J}\}$ appears in $e_{J, J}$ then all the $\{\overline{\sigma J}\}$ are different, where $\sigma \in \mathscr{W}\left(\Psi^{\prime}\right)$. But $\left\{\{\overline{\sigma J}\} \mid \sigma \in \mathscr{W}\left(\Psi^{\prime}\right)\right\}$ is a linearly independent subset of $\left\{\{\overline{d J}\} \mid d \in E_{\boldsymbol{\Psi}}\right\}$. If $e_{J, J^{\prime}}=0$ then $s(\sigma)=(-1)^{l(\sigma)}=0$ for all $\sigma \in \mathscr{W}\left(\Psi^{\prime}\right)$. This is a contradiction and so $e_{J, J^{\prime}} \neq 0$.

Remark 2. In [5] the present author and Morris defined a useful system as a pair $\left\{J, J^{\prime}\right\}$ such that $\mathscr{W}(\Psi) \cap \mathscr{W}\left(\Psi^{\prime}\right)=\langle e\rangle$ and $\mathscr{W}\left(\Psi^{\perp}\right) \cap \mathscr{W}\left(\Psi^{\prime \perp}\right)=\langle e\rangle$. However Lemma 
3.6 and the following lemma show that in this setting the condition $\mathscr{N}(\Psi) \cap \mathscr{W}\left(\Psi^{\prime}\right)=\langle e\rangle$ in our definition of a useful sub-system is necessary.

Lemma 3.8. If there exists $w \in \mathscr{N}(\Psi) \cap \mathscr{W}\left(\Psi^{\prime}\right)$ such that $w$ has order 2 , and $s(w)=-1$ then $e_{J, J^{\prime}}=0$.

Proof. See Lemma 3.14 [5].

Example 3.9. Let $\boldsymbol{\Phi}=\mathbf{G}_{2}$ and $\pi=\left\{\alpha_{1}=\varepsilon_{1}-\varepsilon_{2}, \alpha_{2}=-2 \varepsilon_{1}+\varepsilon_{2}+\varepsilon_{3}\right\}$. Let $\boldsymbol{\Psi}=\mathbf{A}_{1}$ be the subsystem of $\Phi$ with simple system $J=\left\{\alpha_{1}\right\}$ and let $\Psi^{\prime}=\mathbf{A}_{2}$ be the subsystem of $\Phi$ with simple system $J^{\prime}=\left\{\alpha_{2}, 3 \alpha_{1}+\alpha_{2}\right\}$. Thus we have $\mathscr{W}(\Psi)=\left\{e, \tau_{1}\right\}$ and $\mathscr{W}\left(\Psi^{\prime}\right)=$ $\left\{e, \tau_{2}, \tau_{1} \tau_{2} \tau_{1}, \tau_{2} \tau_{1} \tau_{2} \tau_{1}, \tau_{1} \tau_{2} \tau_{1} \tau_{2}, \tau_{2} \tau_{1} \tau_{2} \tau_{1} \tau_{2}\right\}, \mathscr{N}(\Psi)=\left\{e, \tau_{1}, \tau_{2} \tau_{1} \tau_{2} \tau_{1} \tau_{2}, \tau_{2} \tau_{1} \tau_{2} \tau_{1} \tau_{2} \tau_{1}\right\}$. Then $\mathscr{W}(\boldsymbol{\Psi}) \cap \mathscr{W}\left(\boldsymbol{\Psi}^{\prime}\right)=\langle e\rangle$, but $w=\tau_{2} \tau_{1} \tau_{2} \tau_{1} \tau_{2} \in \mathcal{N}(\boldsymbol{\Psi}) \cap \mathscr{W}\left(\boldsymbol{\Psi}^{\prime}\right)$ and $e_{J, J^{\prime}}=0$.

If $\left\{J, J_{1}^{\prime}\right\}$ and $\left\{J, J_{2}^{\prime}\right\}$ are useful sub-systems in $\Phi$ and $\Psi_{1}^{\prime} \subseteq \Psi_{2}^{\prime}$, then $S^{\Psi, \Psi_{2}^{\prime}}$ is a $K \mathscr{W}$-submodule of $S^{\Psi_{.} \Psi_{1}}$, where $J_{1}^{\prime}$ and $J_{2}^{\prime}$ are simple systems for $\Psi_{1}^{\prime}$ and $\Psi_{2}^{\prime}$ respectively.

Now we consider under what conditions $S^{\Psi, \Psi^{\prime}}$ is irreducible.

Lemma 3.10. Let $\left\{J, J^{\prime}\right\}$ be a useful sub-system in $\Phi$ and let $d \in E_{\Psi}$. Then the following conditions are equivalent:

(i) $\{\overline{d J}\}$ appears with non-zero coefficient in $e_{J, J}$;

(ii) there exists $\sigma \in \mathscr{W}\left\{\Psi^{\prime}\right\}$ such that $\sigma\{\bar{J}\}=\{\overline{d J}\}$;

(iii) there exists $\rho \in \mathscr{N}\{\boldsymbol{\Psi}\}$ and $\sigma \in \mathscr{W}\left\{\Psi^{\prime}\right\}$ such that $d=\sigma \rho$.

Proof. This follows from Lemma 3.18 [5].

Lemma 3.11. Let $\left\{J, J^{\prime}\right\}$ be a useful sub-system in $\Phi$ and let $d \in E_{\Psi}$. If $\{\overrightarrow{d J}\}$ appears in $e_{J, J^{\prime}}$ then $d \Psi \cap \Psi^{\prime}=\emptyset$.

Proof. Let $\alpha \in d \Psi$. If $\{\overline{d J}\}$ appears in $e_{J, J}$ then by Lemma $3.10 d=\sigma \rho$, where $\sigma \in \mathscr{W}\left(\Psi^{\prime}\right)$ and $\rho \in \mathcal{N}(\Psi)$. Then $\alpha \in \sigma \rho \Psi$. Since $\rho \in \mathcal{N}(\Psi)$, then $\alpha \in \sigma \Psi$ and $\sigma^{-1} \alpha \in \Psi$. But $\Psi_{\cap} \cap \Psi^{\prime}=\emptyset$, then $\sigma^{-1} \alpha \notin \Psi^{\prime}$. Since $\sigma \in \mathscr{W}\left(\Psi^{\prime}\right), \sigma \Psi^{\prime}=\Psi^{\prime}$ then $\alpha \notin \Psi^{\prime}$.

Lemma 3.12. Let $\left\{J, J^{\prime}\right\}$ be a useful sub-system in $\Phi$ and let $d \in E_{\Psi}$. Let $d \Psi \cap \Psi^{\prime} \neq \emptyset$. Then $\kappa_{J^{\prime}}\{\overline{d J}\}=0$.

Proof. If $d \Psi \cap \Psi^{\prime} \neq \emptyset$ then let $\alpha \in d \Psi \cap \Psi^{\prime}$ and so $\tau_{a} \in \mathcal{N}(d \Psi) \cap \mathscr{W}\left(\Psi^{\prime}\right)$. Thus

$$
\left(e-\tau_{a}\right)\{\overline{d J}\}=\{\overline{d J}\}-\{\overline{d J}\}=0 .
$$

Since $\left\{e, \tau_{a}\right\}$ is a subgroup of $\mathscr{W}\left(\Psi^{\prime}\right)$ then we can select coset representatives $\alpha_{1}, \sigma_{2}, \sigma_{3}, \ldots, \sigma_{s}$ for $\left\{e, \tau_{a}\right\}$ in $\mathscr{W}\left(\Psi^{\prime}\right)$ such that 


$$
\kappa_{J},\{\overline{d J}\}=\left(\sum_{i=1}^{s} \sigma_{i}\right)\left(e-\tau_{\alpha}\right)\{\overline{d J}\}=0
$$

The converse of Lemma 3.11 is not true in general, which leads to the following definition.

Definition 3.13. A useful sub-system $\left\{J, J^{\prime}\right\}$ in $\Phi$ is called a good sub-system if $d \Psi \cap \Psi^{\prime}=\emptyset$ for $d \in E_{\Psi}$ then $\{\overline{d J}\}$ appears with non-zero coefficient in $e_{J, J^{\prime}}$

Lemma 3.14. Let $\left\{J, J^{\prime}\right\}$ be a good sub-system in $\Phi$ and let $d \in E_{\Psi}$.

(i) If $\{\overline{d J}\}$ does not appear in $e_{J, J}$ then $\kappa_{J},\{\overline{d J}\}=0$.

(ii) If $\{\overline{d J}\}$ appears in $e_{J, J}$, then there exists $\sigma \in \mathscr{W}\left(\Psi^{\prime}\right)$ such that

Proof. See Lemma 3.22 [5].

$$
\kappa_{J^{\prime}}\{\overline{d J}\}=s(\sigma) e_{J . J^{\prime}}
$$

By the previous lemma if $M^{\Psi}$, then $\kappa_{J}, m$ is a multiple of $e_{J, J^{\prime}}$.

We now define a bilinear form $\langle$, $\rangle$ on $M^{\Psi}$ by setting

$$
\left\langle\left\{\bar{J}_{1}\right\},\left\{\bar{J}_{2}\right\}\right\rangle= \begin{cases}1 & \text { if }\left\{\bar{J}_{1}\right\}=\left\{\bar{J}_{2}\right\} \\ 0 & \text { otherwise }\end{cases}
$$

This is a symmetric, non-singular, $\mathscr{W}$-invariant bilinear form on $M^{\Psi}$.

Now we can give James' submodule theorem in this new setting.

Theorem 3.15. Let $\left\{J, J^{\prime}\right\}$ be a good sub-system in $\Phi$. Let $U$ be a submodule of $M^{\Psi}$. Then either $S^{\Psi, \Psi^{\prime}} \subseteq U$ or $U \subseteq S^{\Psi, \Psi^{\prime 1}}$, where $S^{\Psi, \Psi^{\prime 1}}$ is the complement of $S^{\boldsymbol{\Psi}, \Psi^{\prime}}$ in $M^{\Psi}$.

Proof. See Theorem 3.24 [5].

We can now prove our principal result.

Theorem 3.16. Let $\left\{J, J^{\prime}\right\}$ be a good sub-system in $\Phi$. The $K \mathscr{W}$-module

$$
D^{\Psi, \Psi^{\prime}}=S^{\Psi, \Psi^{\prime}} / S^{\Psi, \Psi^{\prime}} \cap S^{\Psi, \Psi^{\prime \prime}}
$$

is zero or irreducible.

Proof. If $U$ is a submodule of $S^{\boldsymbol{\Psi}, \boldsymbol{\Psi}^{\prime}}$ then $U$ is a submodule of $M^{\boldsymbol{\Psi}}$ and by Theorem 3.15 either $S^{\Psi, \Psi^{\prime}} \subseteq U$ in which case $U=S^{\Psi, \Psi^{\prime}} \subseteq S^{\Psi, \Psi^{\prime 1}}$ and $U \subseteq S^{\Psi, \Psi^{\prime}} \cap S^{\Psi, \Psi^{\prime 1}}$, which completes the proof. 
Remark 3. Since $E_{\boldsymbol{\Psi}} \subseteq D_{\boldsymbol{\Psi}}$, if $\left\{J, J^{\prime}\right\}$ is a useful sub-system and $S^{\Delta . \Delta^{\prime}}$ is irreducible, then $S^{\mathbf{T}, \boldsymbol{\Psi}^{\prime}}$ is irreducible.

In [5], we see that not all the irreducible modules for $\mathscr{W}\left(\mathbf{D}_{4}\right)$ are obtained by considering subsystems of root systems. In order to obtain additional irreducible character, we define extended subsystems of root systems. It is shown that the irreducible character $\chi_{12}$ of degree 6 is obtained by considering $\mathscr{W}\left(\mathbf{G}_{2}\right)$ as Steinberg subgroup of $\mathscr{W}\left(\mathbf{D}_{4}\right)$.

In the following example we show how the irreducible character $\chi_{12}$ of degree 6 can be obtained by considering a good sub-system and how a good sub-system may be constructed in the Weyl group of type $D_{4}$.

Example 3.17. Let $\Phi=D_{4}$ with simple system

$$
\pi=\left\{\alpha_{1}=\varepsilon_{1}-\varepsilon_{2}, \alpha_{2}=\varepsilon_{2}-\varepsilon_{3}, \alpha_{3}=\varepsilon_{3}-\varepsilon_{4}, \alpha_{4}=\varepsilon_{3}+\varepsilon_{4}\right\}
$$

The representatives of conjugate classes and the character table of $\mathscr{W}\left(\mathbf{D}_{4}\right)$ and the non-conjugate subsystems of $\mathbf{D}_{4}$ have been given in [5].

Let $\boldsymbol{\Psi}=\mathbf{A}_{3}^{\prime}$ be the subsystem of $\mathbf{D}_{4}$ with $J=\{1000,0100,0001\}$. Let $\Psi^{\prime}=A_{1}$ be the subsystem of $\Phi$ which is contained in $\Phi \backslash \Psi$, with simple system $J^{\prime}=\{1110\}$. Then $\mathscr{N}(\Psi)=\mathscr{W}\left(\mathbf{B}_{\mathbf{3}}\right)$ and since $\mathscr{N}(\Psi) \cap \mathscr{W}\left(\Psi^{\prime}\right)=\langle e\rangle$ and $\mathscr{W}\left(\Psi^{\perp}\right) \cap \mathscr{W}\left(\Psi^{\prime 1}\right)=\langle e\rangle$, then $\left\{J, J^{\prime}\right\}$ is a useful sub-system in $\Phi$. Then $\tau_{\Delta^{*}}$ contains the $\Delta^{*}$-tabloids;

$$
\begin{aligned}
& \{\bar{J}\} \quad=\{1000,0100,0001 ; 1110\} \\
& \left\{\tau_{3} \bar{J}\right\}=\{1000,0110,0001 ; 1100\} \\
& \left\{\tau_{2} \tau_{3} \bar{J}\right\}=\{1100,0010,0101 ; 1000\} \\
& \left\{\tau_{1} \tau_{2} \tau_{3} \bar{J}\right\}=\{0100,0010,1101 ;-1000\}
\end{aligned}
$$

For $d=e, \tau_{1} \tau_{2} \tau_{3}$ we have $d \Psi \cap \Psi^{\prime}=\emptyset$. Since

$$
e_{J, J^{\prime}}=\{\bar{J}\}-\left\{\tau_{1} \tau_{2} \tau_{3} \bar{J}\right\}
$$

then $\left\{J, J^{\prime}\right\}$ is a good sub-system in $\boldsymbol{\Phi}$.

Now let $K$ be a field with Char $K=0$. Let $M^{\Psi}$ be $K$-space whose basis elements are the $\Delta^{*}$-tabloids. Let $S^{\Psi, \Psi^{\prime}}$ be the corresponding $K \mathscr{W}$-submodule of $M^{\boldsymbol{\Psi}}$, then by definition of the submodule of Specht module we have

$$
S^{\Psi . \Psi^{\prime}}=\operatorname{Sp}\left\{e_{J, J^{\prime}}, e_{\tau_{3}, J, \tau_{3} J^{\prime}}, e_{\tau_{2} \tau_{3} J_{,} \tau_{2 \tau_{3}} J^{\prime}}\right\}
$$

where

$$
\begin{aligned}
& e_{J . J^{\prime}}=\{\bar{J}\}-\left\{\tau_{1} \tau_{2} \tau_{3} \bar{J}\right\}=e_{\tau_{3} J . \tau_{3} J^{\prime}}-e_{\tau_{2 \tau_{3} J, \tau_{2} \tau_{3} J^{\prime}}} \\
& e_{\tau_{3} J . \tau_{3} J^{\prime}}=\left\{\tau_{3} \bar{J}\right\}-\left\{\tau_{1} \tau_{2} \tau_{3} \bar{J}\right\}=-e_{\tau_{2} \tau_{3} J, \tau_{2} \tau_{3} J^{\prime}} \\
& e_{\tau_{2} \tau_{3} J, \tau_{2} \tau_{3} J^{\prime}}=\left\{\tau_{2} \tau_{3} J\right\}-\left\{\tau_{1} \tau_{2} \tau_{3} \bar{J}\right\}=e_{J, J^{\prime}}-e_{\tau_{3} J, \tau_{3} J^{\prime}}
\end{aligned}
$$


Let $T$ be the matrix representation of $\mathscr{W}$ afforded by $S^{\boldsymbol{\Psi}, \boldsymbol{\Psi}}$ with character $\psi$ and let $\tau_{1} \tau_{3} \tau_{2}$ be the representative of the conjugate class $C_{4}$ as in [5]. Then

$$
\begin{aligned}
& \tau_{1} \tau_{3} \tau_{2}\left(e_{J, J^{\prime}}\right)=\left\{\tau_{3} \bar{J}\right\}-\left\{\tau_{2} \tau_{3} \bar{J}\right\}=e_{t_{3} J, \tau_{3} J^{\prime}}-e_{\tau_{2} \tau_{3} J_{.} \tau_{2 \tau} J^{\prime}} \\
& \tau_{1} \tau_{3} \tau_{2}\left(e_{\tau_{3} J, \tau_{3} J^{\prime}}\right)=\left\{\tau_{1} \tau_{2} \tau_{3} J\right\}-\left\{\tau_{2} \tau_{3} \bar{J}\right\}=-e_{\tau_{2} \tau_{3} J, \tau_{2} \tau_{3} J^{\prime}} \\
& \tau_{1} \tau_{3} \tau_{2}\left(e_{\tau_{2} \tau_{3} J . \tau_{2} \tau_{3} J^{\prime}}\right)=\{\bar{J}\}-\left\{\tau_{2} \tau_{3} \bar{J}\right\}=e_{J, J^{\prime}}-e_{\tau_{3} J . \tau_{3} J^{\prime}}
\end{aligned}
$$

Thus we have

$$
T\left(\tau_{1} \tau_{3} \tau_{2}\right)=\left(\begin{array}{ccc}
0 & 1 & -1 \\
0 & 0 & -1 \\
1 & 0 & -1
\end{array}\right) \text { and } \psi\left(\tau_{1} \tau_{3} \tau_{2}\right)=-1
$$

By a similar calculation to the above it can be showed that $\psi=\chi_{9}$. Now let $\Psi=\mathbf{A}_{2}$ be the subsystem of $\mathbf{D}_{4}$ with $J=\{1000,0100\}$. Let $\Psi^{\prime}=\mathbf{A}_{2}$ be the subsystem of $\Phi$ which is contained in $\boldsymbol{\Phi} \backslash \Psi$, with simple system $J^{\prime}=\{0001,0110\}$. Then $\mathscr{N}(\Psi)=\mathscr{W}\left(\mathbf{G}_{2}\right)$, the corresponding character of $\mathscr{W}$ afforded by $S^{\Psi, \Psi^{\prime}}$ is $\chi_{12}$.

\section{REFERENCES}

1. E. Al-Aamily, A. O. Morris and M. H. Peel The representations of the Weyl groups of type $B_{n}$, J. Algebra 68 (1981), 298-305.

2. R. W. Carter, Conjugacy classes in the Weyl group, Compositio. Math. 25 (1972), 1-59.

3. S. Halicioglu, Specht modules for finite reflection groups, Glasgow Math. J. 37 (1995), 279-287.

4. S. Halicioglu, A basis of Specht modules for Weyl groups, Turkish J. Math. 18 (1994), 311-326.

5. S. Haliciočlu and A. O. Morris, Specht modules for Weyl groups, Contrib. Alg. Geom. 34 (1993), 257-276.

6. G. D. JAMEs, The irreducible representations of the symmetric group, Bull. London Math. Soc. 8 (1976), 229-232.

7. A. O. Morris, Representations of Weyl groups over an arbitrary field, Astérisque 87-88 (1981), 267-287.

8. W. SРеСнт, Die irreduziblen Darstellungen der symmetrischen Gruppen, Math. Z. 39 (1935), 679-711.

Department of Mathematics

ANKARA UNIVERSITY

06100 TaNDOGan

ANKARA

TURKEY 